\title{
On the Optimal Number of Hops in Linear Wireless Ad Hoc Networks with Hybrid ARQ
}

\author{
Igor Stanojev, Osvaldo Simeone and Yeheskel Bar-Ness \\ Center for Wireless Communications and Signal Processing \\ New Jersey Institute of Technology, Newark, New Jersey 07102-1982, USA \\ e-mail: \{iss2, osvaldo.simeone, yeheskel.barness\}@njit.edu
}

\begin{abstract}
In this paper, we study the optimal design of a linear wireless multihop network that employs Hybrid Automatic Repeat reQuest (HARQ) protocols in a quasi-static fading environment. Data transmission is assumed to be delay-tolerant up to a certain predefined maximum delay (in transmission slots) that is larger or equal to the number of hops. Multihop routing is implemented through a time-division scheduling and decode-andforward relaying. Excess time is exploited for retransmissions using either HARQ Type I or Chase Combining protocols on the hops that result in link-outage. Relying on analysis and numerical optimization, we seek answer to the following question: given the maximum allowed delay and signal-to-noise ratio, what is the optimal number of hops that maximizes the end-to-end throughput?
\end{abstract}

\section{INTRODUCTION}

Characterized by low-powered single-antena terminals and often lacking any supporting infrastructure, ad-hoc networks typically operate in the low signal-to-noise ratio (SNR) regime and, in general, need to rely on cooperation between terminals in order to cover broader regions [1]. The simplest cooperative transmission approach is multihopping, whereby a packet is sequentially routed from source to destination through a series of hops. For a given source-destination pair, the optimal topology for a multihop network generally corresponds to a linear network, wherein the terminals are equidistantly placed on the line between source and destination. This network, although rarely encountered in practice and considered to be rather optimistic, allows for tractable analysis and for the establishment of important insights into the performance of general multihop networks [2].

Multihop transmission for a linear ad-hoc network was investigated in [2], where it was demonstrated that multihop, both with or without spatial reuse ${ }^{1}$, is advantageous (in terms of power efficiency) in the low-SNR regime, thanks to power gains or low interference (compared to the noise level), but fails for large values of SNR, due to interference limitations or throughput reduction (as a consequence of multiple transmissions). Approximate analytical solution for the optimal number of hops in such a network, but without spatial reuse, is given in [3] by the same authors. The analysis of [2] and [3] is limited to Gaussian (unfaded) channels. In [4],

\footnotetext{
${ }^{1}$ Spatial reuse refers to a multihop scheme whereby the terminals are allowed to transmit simultaneously, so as to increase the end-to-end throughput. For a network with terminals operating in a half duplex regime, neighboring terminals are not allowed to transmit at the same time.
}

a linear multihop network with no spatial reuse is analyzed under a quasi-static fading assumption: an upper bound on the probability of outage is found and exploited, along with the result of [3], to determine the optimal number of hops. Finally, in [5], the authors discuss the impact of various classes of per-hop memoryless retransmission protocols on linear multihop network performance. Namely, the main goal of [5] is to determine the statistics of the overall number of per-hop transmissions in the system, based on a Gilbert-Elliot model for the wireless channels.

In this work, we adopt the same model of a linear multihop network as in [2]-[4]. Specifically, we consider quasi-static fading without spatial reuse, as in [4], and extend the analysis therein by including Hybrid Automatic Repeat reQuest (HARQ) retransmission protocols. We note that, with respect to [5], our setting differs in terms of a wireless channel model (quasi-static Rayleigh instead of Gilbert-Elliot of [5]); more importantly, unlike [5], here we focus on the problem of optimal network design along the lines of [2]-[4]. In particular, we seek answer to the following question: for a linear network with a given maximum tolerated delay, what is the optimal number of hops (i.e., relays) that achieves the maximum end-to-end throughput? Analytical framework is provided for setting of the optimization problem, while the problem itself is solved using numerical methods. Two HARQ protocols are considered, namely HARQ Type I (HARQ-TI) protocol, that prescribes memoryless detection (i.e., erroneous packets are discarded at the receivers), and Chase Combining protocol (HARQ-CC), whereby erroneous packets at the destination are preserved for soft combing with the currently received packet. It is interesting to notice that the results obtained in this work qualitatively confirm the main conclusions of [2]-[4] (e.g., relative multihop gain in low- and high-SNR regimes), notwithstanding the differences in the underlying models, namely, the use of HARQ protocols in this paper to cope with quasi-static fading channels.

\section{System ANALYSIS}

\section{A. System Model}

Consider a linear $k$-hop wireless network, consisting of the source $\mathrm{N}_{1}$, destination $\mathrm{N}_{k+1}$ and $k-1$ relays, $\mathrm{N}_{2}, . ., \mathrm{N}_{k}$, equidistantly placed on the line connecting $\mathrm{N}_{1}$ and $\mathrm{N}_{k+1}$, as depicted in fig. 1. The nodes operate in half-duplex, and only one node can transmit at a given slot, i.e., no spatial reuse 


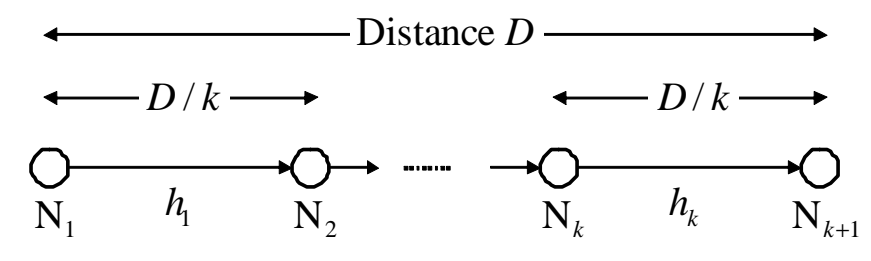

Fig. 1. Linear wireless $k$-hop network.

is allowed. Thus, a packet originating from the source and intended for the destination is routed through each of the $k$ hops in a separate time-slot and the successful transmission requires at least $k$ time-slots. Furthermore, the overall delay tolerated by the network, measured in transmission slots, is $L \geq k$. The additional $L-k$ transmissions slots are used (if necessary) for retransmissions via HARQ-TI or HARQ-CC protocols on the hops that failed to support successful packet delivery. The channel gains $h_{i}^{(t)}$, where $i$ is the number of the hop and $t$ is the number of the retransmission attempt (including the original transmission, $t=1$ ) on that hop, are modelled as independent proper complex Gaussian random variables with unit power. That is, the model assumes Rayleigh block-fading, whereby the channel gain is constant during a slot but changes independently with each slot. All nodes transmit with equal power, and the signal-to-noise ratio of the single-hop system $(k=1)$ is SNR. Denoting the path-loss exponent as $\eta$, the signal-to-noise ratio on any of the hops of a $k$-hop network is then $\mathrm{SNR}_{k}=\mathrm{SNR} \cdot k^{\eta}$.

\section{B. Analysis}

As stated earlier, our goal is to determine the optimal number of hops $k$, given the maximum delay $L$ and SNR. As the performance criterion, we employ the end-to-end throughput, defined as the average number of successfully transmitted bits per slot. This section is largely dedicated to the derivation of the throughput and its relation to parameters $k, L$ and SNR. Once this relation is established, we discuss the problem of solving for optimal $k$, i.e., $k_{\text {opt }}(L, \mathrm{SNR})$, in Sec. II-C.

Using renewal theory, the overall throughput $R$ can be shown to be (irrespective of the employed HARQ protocol) [6]:

$$
R=\frac{r\left(1-P_{\text {out }}(L)\right)}{\bar{l}},
$$

where $r$ is the rate (in bit/s/Hz) of the original transmission (transmission rate); $P_{\text {out }}(L)$ is the probability of outage after $L$ slots, i.e., the probability that after $L$ slots the destination node $\mathrm{N}_{k+1}$ still did not successfully decode the packet; and $\bar{l}$ is the average number of slots per packet used for end-toend transmission, $k \leq \bar{l} \leq L$. It is noted that a packet is dropped (i.e., outage event occurs) if the maximum number of retransmissions $L$ is reached and the packet is still not correctly decoded.
The average number of slots $\bar{l}$ in (1) can be written as

$$
\begin{aligned}
\bar{l}= & k+\sum_{l=k}^{L}(l-k)\left[P_{\text {out }}(l-1)-P_{\text {out }}(l)\right] \\
& +(L-k) P_{\text {out }}(L) \\
= & k+\sum_{l=k}^{L-1} P_{\text {out }}(l),
\end{aligned}
$$

where we exploited the fact that $P_{\text {out }}(l-1)-P_{\text {out }}(l)$ is the probability of the successful end-to-end transmission achieved after exactly $l$ slots [6]. Using the previous equation, the endto-end throughput $R$ in (1) can be conveniently expanded as:

$$
R=r \frac{1-P_{\text {out }}(L)}{k+\sum_{l=k}^{L-1} P_{\text {out }}(l)} .
$$

In order to derive the probability of outage after $l$ transmission slots, $P_{\text {out }}(l)$, we first define $p_{S}\left(a_{i}\right)$ as the probability that the $i$ th hop delivers successfully a packet from the node $\mathrm{N}_{i}$ to the node $\mathrm{N}_{i+1}$ after exactly $a_{i} \geq 1$ (integer) slots. Denoting $\sum_{\mathcal{A}_{j}}(\cdot)$ as the summation over all the tuples in the set $\mathcal{A}_{j}=\left\{\left(a_{1}, . ., a_{k}\right) \mid a_{i} \in \mathcal{N}, a_{1}+. .+a_{k}=j\right\}$, then $\sum_{\mathcal{A}_{j}} \prod_{i=1}^{k} p_{S}\left(a_{i}\right)$ is the probability of successful end-to-end transmission after a total delay of exactly $j$ slots. Furthermore, since the outage after $l$ transmission slots excludes all events that would lead to successful transmission within $l$ slots (i.e., $j=k, . ., l)$, the probability of outage $P_{\text {out }}(l)$ can be written as:

$$
P_{\text {out }}(l)=1-\sum_{j=k}^{l} \sum_{\mathcal{A}_{j}} \prod_{i=1}^{k} p_{S}\left(a_{i}\right) .
$$

Furthermore, the probability $p_{S}\left(a_{i}\right)$ can also be expressed in terms of the probability of unsuccessful transmission in the $i$ th hop after $a_{i}$ slots, $p_{e}\left(a_{i}\right)$ [6]:

$$
p_{S}\left(a_{i}\right)=p_{e}\left(a_{i}-1\right)-p_{e}\left(a_{i}\right) .
$$

Notice that, in order to calculate the throughput $R$ in (3), according to (4) and (5), it suffices to calculate the probability of unsuccessful transmission in the $i$ th hop after $a_{i}$ transmission slots, $p_{e}\left(a_{i}\right)$, which depends on employed HARQ protocols. Applying the information-theoretic setting as in, e.g., [6], the event of unsuccessful per-hop transmission after $a_{i}$ transmission slots is defined as the event wherein the rate achievable after $a_{i}$ transmissions on the $i$ th hop is smaller than the transmission rate $r$. The following two subsections, Sec. IIB.1 and Sec. II-B.2, are dedicated to the throughput derivation for HARQ-TI and HARQ-CC protocols, respectively.

1) HARQ-TI: Since the HARQ-TI protocol is memoryless, i.e., the erroneously received packets are dropped, the probability $p_{e}\left(a_{i}\right)$ for HARQ-TI can be written as:

$$
\begin{aligned}
p_{e, T I}\left(a_{i}\right) & =\operatorname{Pr}\left\{r>\max _{t=1, ., a_{i}} \log _{2}\left(1+\left|h_{i}^{(t)}\right|^{2} \mathrm{SNR}_{k}\right)\right\} \\
& =\operatorname{Pr}\left\{\max _{t=1, \ldots, a_{i}}\left|h_{i}^{(t)}\right|^{2}<\frac{2^{r}-1}{\mathrm{SNR}_{k}}\right\} \\
& =\operatorname{Pr}\left\{\left|h_{i}^{(t)}\right|^{2}<\frac{2^{r}-1}{\mathrm{SNR}_{k}}\right\}^{a_{i}}
\end{aligned}
$$


Notice that in (6) we assumed that the terminals are using capacity-achieving Gaussian codebooks. Recalling that $h_{i}^{(t)}$ is a complex Gaussian random variable, it follows that $\left|h_{i}^{(t)}\right|^{2}$ is an exponentially distributed random variable, and, finally, $p_{e, T I}\left(a_{i}\right)$ becomes:

$$
p_{e, T I}\left(a_{i}\right)=\left(1-e^{-\mu(r, k, \mathrm{SNR})}\right)^{a_{i}}
$$

where

$$
\mu(r, k, \mathrm{SNR})=\frac{2^{r}-1}{2 \mathrm{SNR} \cdot k^{\eta}} .
$$

In the following, we drop the dependence of $\mu$ on $r, k$ and SNR, for notation convenience.

Applying (7) to (5), we find

$$
p_{S, T I}\left(a_{i}\right)=e^{-\mu}\left(1-e^{-\mu}\right)^{a_{i}-1} .
$$

Then, using (4), we have that the probability of outage after $l$ slots, $P_{\text {out }}(l)$, reads:

$$
\begin{aligned}
P_{\text {out }, T I}(l) & =1-\sum_{j=k}^{l} \sum_{\mathcal{A}_{j}} \prod_{i=1}^{k} p_{S, T I}\left(a_{i}\right) \\
& =1-\sum_{j=k}^{l} \sum_{\mathcal{A}_{j}} e^{-k \mu}\left(1-e^{-\mu}\right)^{j-k},
\end{aligned}
$$

where we used the fact that, for the set $\mathcal{A}_{j}, \sum_{i=1}^{k} a_{i}=j$. Furthermore, as shown in the Appendix, the cardinality of set $\mathcal{A}_{j}$ is $\alpha_{j}=\left|\mathcal{A}_{j}\right|=\left(\begin{array}{c}j-1 \\ k-1\end{array}\right)$. Then, (10) can be further simplified as

$$
\begin{aligned}
P_{\text {out }, T I}(l) & =1-\sum_{j=k}^{l} \alpha_{j} e^{-k \mu}\left(1-e^{-\mu}\right)^{j-k} \\
& =1-e^{-k \mu} \sum_{j=0}^{l-k} \alpha_{j+k}\left(1-e^{-\mu}\right)^{j} .
\end{aligned}
$$

With (11), the denominator of (3), i.e., the average number of exploited transmission slots per packet $\bar{l}$ (2), for the HARQ-TI protocol, is easily shown to be:

$$
\begin{aligned}
\bar{l}_{T I} & =k+\sum_{l=k}^{L-1} P_{\text {out }}(l) \\
& =L-e^{-k \mu} \sum_{l=k}^{L-1} \sum_{j=0}^{l-k} \alpha_{j+k}\left(1-e^{-\mu}\right)^{j} \\
& =L-e^{-k \mu} \sum_{l=0}^{L-k-1} \alpha_{l+k}(L-k-l)\left(1-e^{-\mu}\right)^{l} .
\end{aligned}
$$

Finally, applying (11) and (12) to (3), the throughput for HARQ-TI protocol reads:

$$
R_{\mathrm{TI}}=r \frac{e^{-k \mu} \sum_{l=0}^{L-k} \alpha_{l+k}\left(1-e^{-\mu}\right)^{l}}{L-e^{-k \mu} \sum_{l=0}^{L-k-1} \alpha_{l+k}(L-l-k)\left(1-e^{-\mu}\right)^{l}} .
$$

2) HARQ-CC: For HARQ-CC protocol, the previously received erroneous packets are preserved and soft-combined at the receiver with the currently received packet, and the probability $p_{e}\left(a_{i}\right)$ for HARQ-CC protocol reads:

$$
\begin{aligned}
p_{e, C C}\left(a_{i}\right) & =\operatorname{Pr}\left\{r>\log _{2}\left(1+\sum_{t=1}^{a_{i}}\left|h_{i}^{(t)}\right|^{2} \mathrm{SNR}_{k}\right)\right\} \\
& =\operatorname{Pr}\left\{\sum_{t=1}^{a_{i}}\left|h_{i}^{(t)}\right|^{2}<\frac{2^{r}-1}{\mathrm{SNR}_{k}}\right\} \\
& =F\left(2 \mu, 2 a_{i}\right)
\end{aligned}
$$

where $F(x, \nu)$ is a cumulative distribution function of a chisquare random variable taken at value $x$ with $\nu$ degrees of freedom, and $\mu$ was defined in (8). Notice that in (14) we exploited the fact that $\sum_{t=1}^{a_{i}}\left|h_{i}^{(t)}\right|^{2}$ is a chi-square random variable with $2 a_{i}$ degrees of freedom. Recalling that $a_{i}$ is an integer, the probability $p_{e, C C}\left(a_{i}\right)$ can also be written as [7]:

$$
\begin{aligned}
p_{e, C C}\left(a_{i}\right) & =F\left(2 \mu, 2 a_{i}\right)=\frac{\gamma\left(a_{i}, \mu\right)}{\Gamma\left(a_{i}\right)} \\
& =1-e^{-\mu} \sum_{j=0}^{a_{i}-1} \frac{\mu^{j}}{j !},
\end{aligned}
$$

where we exploited the fact that for an integer index $a_{i}$, the incomplete and the ordinary Gamma functions are conveniently expressed as $\gamma\left(a_{i}, \mu\right)=\left(a_{i}-1\right) !\left(1-e^{-\mu} \sum_{j=0}^{a_{i}-1} \frac{\mu^{j}}{j !}\right)$ and $\Gamma\left(a_{i}\right)=\left(a_{i}-1\right)$ !, respectively.

Using (15) in (5), we find:

$$
p_{S, C C}\left(a_{i}\right)=e^{-\mu} \frac{\mu^{a_{i}-1}}{\left(a_{i}-1\right) !} .
$$

Then, using (4), we have:

$$
\begin{aligned}
P_{\text {out }, C C}(l) & =1-\sum_{j=k}^{l} \sum_{\mathcal{A}_{j}} \prod_{i=1}^{k} p_{S, C C}\left(a_{i}\right) \\
& =1-\sum_{j=k}^{l} \sum_{\mathcal{A}_{j}} e^{-k \mu} \frac{\mu^{j-k}}{\prod_{i=1}^{k}\left(a_{i}-1\right) !} \\
& =1-e^{-k \mu} \sum_{j=0}^{l-k} \beta_{j+k} \mu^{j},
\end{aligned}
$$

where $\beta_{l}=\sum_{\mathcal{A}_{j}} \prod_{i=1}^{k} \frac{1}{\left(a_{i}-1\right) !}$. Furthermore, (17) can be exploited to determine the denominator of (3), i.e., the average number of slots per packet, $\bar{l}(2)$, for the HARQ-CC protocol:

$$
\begin{aligned}
\bar{l}_{C C} & =k+\sum_{l=k}^{L-1} P_{\text {out }, C C}(l) \\
& =L-e^{-k \mu} \sum_{l=k}^{L-1} \sum_{j=0}^{l-k} \beta_{j+k} \mu^{j} \\
& =L-e^{-k \mu} \sum_{l=0}^{L-k-1} \beta_{l+k}(L-k-l) \mu^{l} .
\end{aligned}
$$


Applying (17) and (18) to (3), the throughput $R$ for HARQCC protocol finally reads:

$$
R_{\mathrm{CC}}=r \frac{e^{-k \mu} \sum_{l=0}^{L-k} \beta_{l+k} \mu^{l}}{L-e^{-k \mu} \sum_{l=0}^{L-k-1} \beta_{l+k}(L-l-k) \mu^{l}} .
$$

\section{Discussion on System Design}

As discussed in [6], [8]-[9], in order for HARQ protocols to reach their full potential, an optimal choice of the rate $r$ (i.e., transmission rate) for the given SNR, is mandatory. This is even more relevant for multihop systems that can exploit the increase in per-hop signal-to-noise ratio $\left(\mathrm{SNR}_{k}=\mathrm{SNR} \cdot k^{\eta}\right)$ by increasing their transmission rate so as to compensate for the throughput reduction due to the $k$ transmissions and the absence of spatial reuse. The optimization of rate $R$ in (13) and (19) over transmission rate $r$ can be stated as:

$$
r_{\text {opt }}(k, L, \mathrm{SNR})=\arg \max _{r \geq 0} R(r, k, L, \mathrm{SNR}) .
$$

The solution to this problem demands, to the best of our knowledge, numerical methods for global optimization; fortunately, (20) is a one-dimensional problem and, therefore, relatively easily solved. Interested reader is referred to [6], [8][9], wherein the authors present the analysis for the optimal rate $r$ in single-hop networks using the HARQ protocols, under different assumptions and optimization goals.

Having obtained $r_{\text {opt }}$ (20), the following and final step of our optimization process is to determine the optimal number of hops (relays),

$$
k_{\text {opt }}=\arg \max _{k=1,2, . . L} R\left(r_{o p t}(k, L, \mathrm{SNR}), k, L, \mathrm{SNR}\right) .
$$

The problem (21) belongs to the integer programming class. We note, however, that one can approach it using an exhaustive search over $k \in\{1,2, . ., L\}$. Namely, since the maximum delay $L$ is, in practice, rarely expected to be large, a brute force optimization (21) would in general be acceptable in terms of memory and processing demands.

\section{NUMERICAL EXAMPLES}

As explained in the previous section, the optimization process exemplified by (20)-(21) is hardly tractable analytically. In order to get a further insight into the system behavior and understand the properties of optimal design, we resort in this section to numerical results.

Fig. 2 aims at illustrating the optimization problem (20) by presenting the throughput $R$ versus the transmission rate $r$, for a fixed delay $L=14,1 \leq k \leq 12$, SNR $=-10 \mathrm{~dB}$ and (as used throughout this section, unless explicitly mentioned otherwise) $\eta=3$ and HARQ-CC protocol (19). It can be seen from this figure that, for given $k, L$ and $\mathrm{SNR}$, the throughput $R$ has a quasi-concave shape as a function of rate $r$ and a global maximum. In particular, as it also follows from (3), the throughput (for a given $k$ ) increases with the transmission rate $r$, but only up to a point (given by $r_{o p t}(20)$ ) when the negative impact on the probability of outage $P_{\text {out }}(l)$ becomes

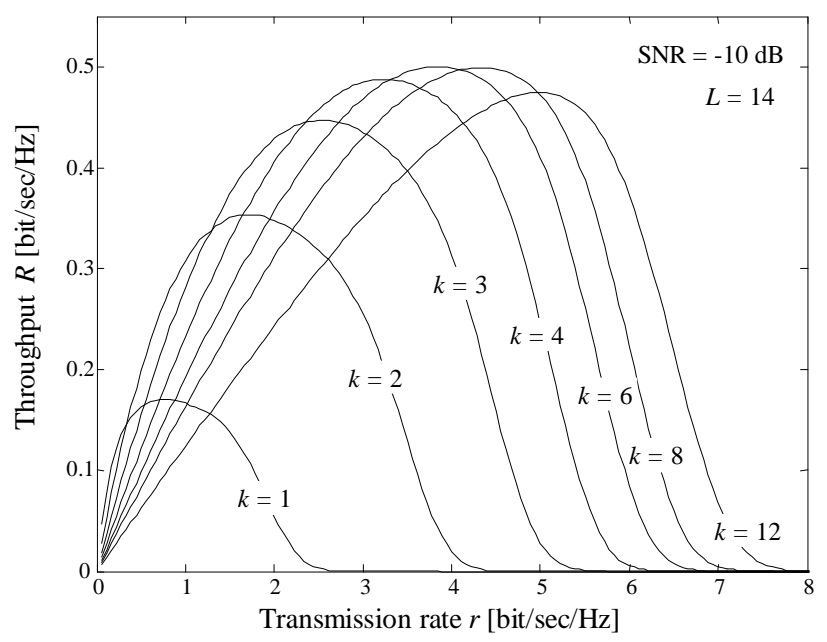

Fig. 2. HARQ-CC: Throughput $R$ versus transmission rate $r$ and number of hops $k$, for the delay $L=14$ and SNR $=-10 \mathrm{~dB}$.

dominant. Furthermore, note that the optimal transmission rate $r_{o p t}$ increases with an increase of the number of hops, due to the enlargement of effective SNR per hop, $\mathrm{SNR}_{k}$.

Having obtained $r_{\text {opt }}$ in (20), the next step is the optimization over the number of hops (21). Fig. 2 already depicts one important property of this step: the optimized throughput (i.e., the throughput maximized over $r$ ) increases with $k$ (i.e., with $\mathrm{SNR}_{k}$ ) up to a point, given by $k_{\text {opt }}$, when the number of hops becomes a limiting factor for the throughput. To further shed light on properties of (21), fig. 3 shows the system throughput versus SNR for fixed $L=4,1 \leq k \leq 3$ and optimized transmission rate $r_{\text {opt }}$. In fact, a larger number of hops is mostly preferable in the low-SNR region, where the benefit of the effective SNR increase of $k^{\eta}$ times is particularly important ${ }^{2}$. However, performance of multihop schemes for larger SNR falls behind the single-hop scheme, as the rate becomes limited by the number of hops, and the retransmission protocols become preferable. Notice that these conclusions (obtained through numerical results) are similar to the results in [2], based on a Gaussian (unfaded) model.

Based on fig. 3, fig. 4 aims at concluding our discussion on the optimization (21), by determining the optimal number of hops $k_{\text {opt }}$, for a given delay $L$ and SNR. It shows that, as the SNR decreases, the system tends to increase the number of hops and, for extremely low SNR, the maximum delay is fully exploited for multihopping, $\lim _{\mathrm{SNR} \rightarrow 0} k_{\text {opt }}=L^{3}$. Furthermore, note that for any SNR value (visible for SNR> $-30 \mathrm{~dB}$ in fig.4), there exists an upper limit on the optimal number of relays that can improve the system performance, even if an infinite delay is allowed.

\footnotetext{
${ }^{2}$ It is also obvious that the larger values of propagation-loss exponent $\eta$ would exercise a positive influence on multihop scheme.

${ }^{3}$ Notice that the delay values (i.e., $L$ ) in fig. 4 are extremely large and rarely encountered in practice. However, while the results would not be qualitatively altered for lower values of $L$, the choice of $L$ in fig. 4 is convenient for description of delay-unconstrained system.
} 


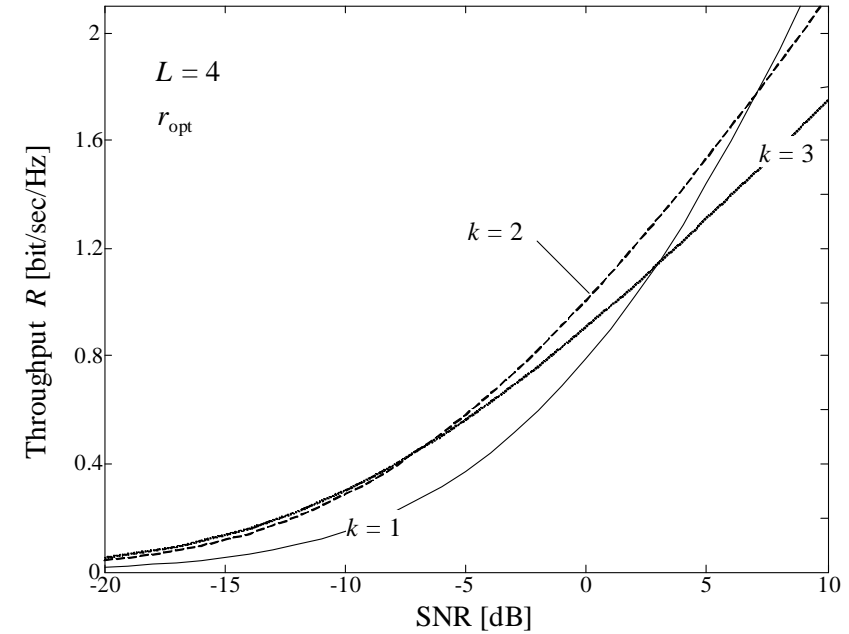

Fig. 3. HARQ-CC: Throughput $R$ versus SNR, for $L=4, k=1 \div 3$ and optimized transmission rate $r_{\text {opt }}(k, L, \mathrm{SNR})$.

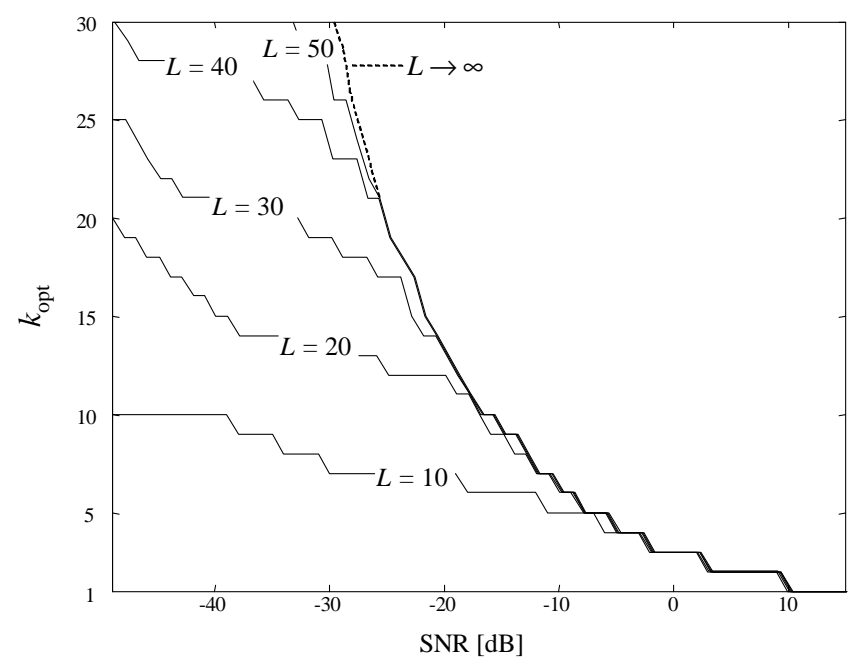

Fig. 4. HARQ-CC: Optimized number of hops $k_{o p t}$ versus SNR and delay L.

Figures 2-4 described the optimization process and the properties of optimal parameters $r_{o p t}$ and $k_{o p t}$. The throughput of optimally designed system, i.e., the system employing $r_{\text {opt }}$ and $k_{\text {opt }}$, is shown in fig. 5, as a function of SNR and delay $(L=4,12)$. For comparison, fig. 5 also shows the "pure" HARQ-CC (single-hop) and "pure" multihop (no retransmissions) schemes employing $L=4,12$ and $k=4,12$ slots, respectively, and a single hop system with no delay $(L=1)$. The figure confirms that the "pure" HARQ-CC and "pure" multihop schemes perform poorly in low and high-SNR region, respectively; however, by exploiting both approaches (multihop-HARQ), the system throughput will increase for a broad SNR region.

Finally, fig. 6 compares the throughput of optimized schemes employing the multihopping with HARQ-TI and HARQ-CC protocols. As expected, the scheme employing

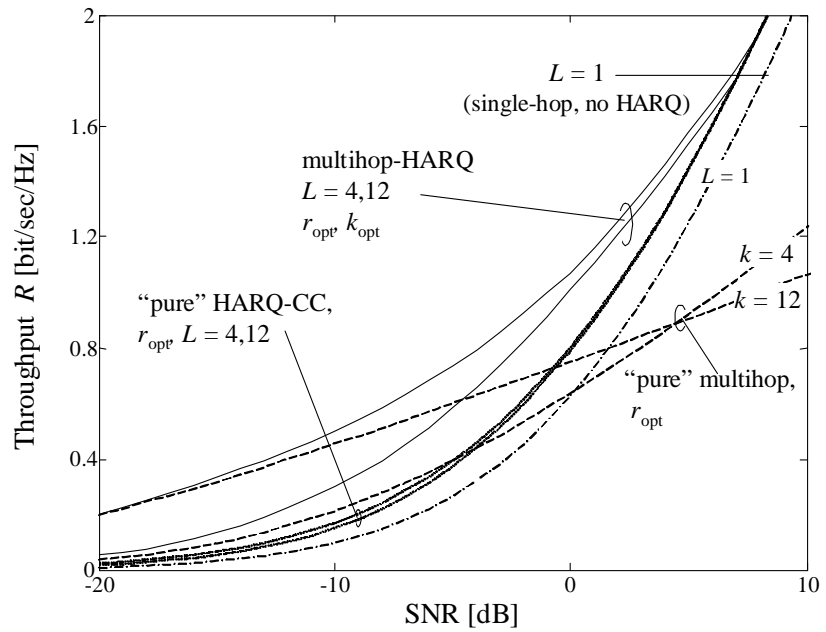

Fig. 5. HARQ-CC: Throughput $R$ versus SNR and delay $L$, for optimized transmission rate $r_{o p t}$, and number of hops $k_{\text {opt }}$.

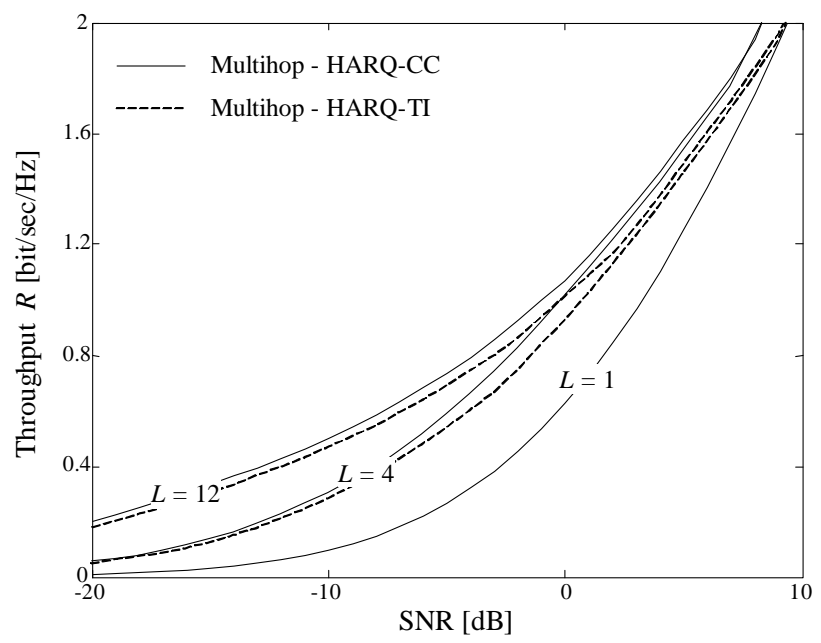

Fig. 6. Throughput $R$ versus SNR and delay $L$, for HARQ-TI and HARQ$\mathrm{CC}$, optimized transmission rate $r_{\text {opt }}$ and number of hops $k_{\text {opt }}$.

the memoryless HARQ-TI is outperformed by the scheme exploiting the HARQ-CC protocol. Notice that the difference between two schemes is relatively constant over a broad SNR region. This behavior is quite different than the behavior of "pure" HARQ schemes, wherein the difference between the two protocols is more emphasized in region where SNR is low. Namely, as the SNR decreases, the (optimized) network relies heavily on the multiple hops, rather than on retransmissions, and the memory of HARQ-CC protocol cannot achieve the full advantage. Optimization of the transmission rate $r$ influences the system in a similar manner, as it prevents a need for a large number of retransmissions, which again reduces the advantage of HARQ-CC protocol.

\section{CONClusions}

In this work, we investigated the optimal linear network design, i.e., the number of employed hops/relays that maximizes 
end-to-end throughput, for a delay-tolerant (up to a given maximum delay) system employing both multihop and HARQ$\mathrm{TI} / \mathrm{CC}$ protocols. It was determined by numerical examples that an optimally designed system exploits the delay primarily through multihopping and HARQ protocols in low and highSNR region, respectively; the relatively good performance of multihop in low-SNR region (and its relatively poor performance in high-SNR region) confirms the analytical results of [3], that were limited to Gaussian (unfaded) channels. Furthermore, we also observed that for relatively large values of SNR, even if infinite delay is allowed, there is an upper limit on the optimal number of relays that would result in further throughput increase. Finally, the results showed that the system employing both multihop and HARQ, if optimally designed, significantly benefits from the allowed delay, as compared to the systems employing only multihop or HARQ protocols.

\section{APPENDIX}

\section{Cardinality of Set $\mathcal{A}_{j}$}

Recall that the set $\mathcal{A}_{j}$ is defined as

$$
\mathcal{A}_{j}=\left\{\left(a_{1}, . ., a_{k}\right) \mid a_{i}=1,2, . . ; a_{1}+. .+a_{k}=j\right\} .
$$

The cardinality of such a set is equal to the number of solutions $\left(a_{1}, . ., a_{k}\right)$ of the following equality:

$$
a_{1}+\ldots+a_{k}=j,
$$

where $a_{1}, . ., a_{k}$ are positive integers. Notice that this problem is equivalent to placing $k-1$ objects of one type between

$j$ sequentially placed objects of another type. The number of solutions of such a problem and, therefore, the cardinality of set $\mathcal{A}_{j}$, is then

$$
\left|\mathcal{A}_{j}\right|=\left(\begin{array}{l}
j-1 \\
k-1
\end{array}\right)
$$

\section{REFERENCES}

[1] A. J. Goldsmith and S. B. Wicker, "Design Challenges for EnergyConstrained Ad Hoc Wireless Networks", IEEE Wireless Commun. Mag., vol. 9, no. 4, pp. 8-27, Aug. 2002.

[2] M. Sikora, J. N. Laneman, M. Haenggi, D. J. Costello and T. E. Fuja, "Bandwidth- and power-efficient routing in linear wireless networks", IEEE Trans. Inf. Theory, vol. 52, no. 6, pp. 2624-2633, Jun. 2006.

[3] M. Sikora, J. N. Laneman, M. Haenggi, D. J. Costello and T. E. Fuja, "On the optimum number of hops in linear wireless networks", in Proc. IEEE ITW, pp. 165-169, 2004.

[4] Ö. Oyman and S. Sandhu, "A Shannon-Theoretic Perspective on Fading Multihop Networks", pp. 525-530, in Proc. IEEE CISS, 2006.

[5] T. Issariyakul and E. Hossain, "Performance Modeling and Analysis of a Class of ARQ Protocols in Multi-Hop Wireless Networks", IEEE Trans. Wireless Commun., vol. 5, no. 12, pp. 3460-3468, Dec. 2006.

[6] G. Caire and D. Tuninetti, "The Throughput of Hybrid-ARQ Protocols for the Gaussian Collision Channel", IEEE Trans. Inf. Theory, vol. 47, no. 5, pp. 1971-1988, Jul. 2001.

[7] M. Abramowitz and I. A. Stegun, Handbook of Mathematical Functions with Formulas, Graphs, and Mathematical Tables, Dover, 1972.

[8] I. Bettesh and S. Shamai, "Optimal power and rate control for minimal average delay: the single-user case", IEEE Trans. Inf. Theory, vol. 52, no. 9, pp. 4115-4141, Sep. 2006.

[9] Q. Liu, S. Zhou, and G. B. Giannakis, "Cross-layer combining of adaptive modulation and coding with truncated ARQ over wireless links," IEEE Trans. Wireless Commun., vol 3, no. 5, pp. 1746-1755, Sep. 2004. 